\title{
Importancia del plan estratégico en la gestión bibliotecaria en beneficio de los usuarios y la biblioteca
}

\section{Importance of the strategic plan in library management for the benefit of users and the library}

\author{
Cesar Olguin-Camacho \\ cesarenba@gmail.com \\ Universidad Nacional Autónoma de México
}

\section{Resumen}

Este documento presenta una propuesta general sobre cómo desarrollar un plan estratégico que apoye a los gerentes de bibliotecas en su preparación y así poder establecer el curso en el que se desea que trabaje la biblioteca. Para una mejor comprensión de esto, se divide en pasos que guiarán su conformación.

\section{Palabras clave}

Administración de bibliotecas, Plan estratégico, Gestión bibliotecaria, Usuarios.

\begin{abstract}
This paper presents a general proposal on how to develop a strategic plan that supports library managers in its preparation and thus be able to set the course in which the library is desired to work. For a better understanding of this, it is divided into steps that will guide its conformation.
\end{abstract}

\section{Keywords}

Library administration, Strategic plan, Library management, Users. 
Recibido: 21/08/2019

Aceptado: 19/10/2019

DOI: https://dx.doi.org/10.5557/IIMEI10-N19-055074

Descripción propuesta: Olguin-Camacho, Cesar. Importancia del plan estratégico en la gestión bibliotecaria en beneficio de los usuarios y la biblioteca Métodos de Información, 10(19), 55-74

\section{Introducción}

En la gestión bibliotecaria hoy en día es más dinámica, al igual que las fuentes de información y los usuarios estos últimos han cambiado sus hábitos de información frente a la biblioteca, gracias a los avances tecnológicos en las nuevas tendencias de la información y el conocimiento. Los responsables y los usuarios son la columna vertebral de la biblioteca, por lo cual se deben tomar en cuenta ambas partes, para que la biblioteca cumpla su función de satisfacer las necesidades de los usuarios, mediante la elaboración de un plan estratégico que cumpla con los objetivos y metas que persigue la biblioteca en beneficio de su comunidad.

Los servicios que ofrece la biblioteca están basados en las necesidades de información de los usuarios, con el fin de satisfacerlos. Para ello la planeación de las actividades y mejoramiento de cada servicio, no deben darse de manera inferida, al azar o por moda. Para ello es vital elaborar un plan estratégico basado en la gestión bibliotecaria y en un estudio de usuarios, que plasme de antemano lo que deseamos lograr y cómo llevarlo a cabo.

El plan estratégico que se diseñe para alcanzar la misión de la biblioteca debe alinearse al plan de la institucional, al que nos encontremos subordinados, este debe elaborarse de manera minuciosa pensando siempre en el bienestar de la biblioteca y del usuario de nuestra comunidad.

\section{Fundamentos}

La planeación o planificación como suele llamarse también es el primer paso dentro del proceso administrativo, reflejándose en la gestión bibliotecaria, es aquí donde se elaboran los diferentes planes a realizar dentro del tiempo en 
que un coordinador, director o responsable esté al frente de la biblioteca, centro de información o unidad de información.

Dentro de las fases de la administración se encuentra la planeación, la cual es la columna vertebral de las subbases (Terry George 1971): organización, dirección y control, es decir, aplicando estos principios a la biblioteca se establecerá, el qué hacer, cómo hacerlo, cuándo hacerlo y quien ha de hacerlo, estos elementos nos guiaran donde estamos y hasta donde queremos ir, teniendo claramente el objetivo que se persigue y cómo se logrará, para ello se tienen que establecer los diferentes elementos que darán sentido a nuestro existir como unidad de información. Aquí se marca la misión de la biblioteca, los objetivos generales, elaboración de proyectos a emprender tanto los fijos como aquellos que se darán por única vez, evitando una desorganización de actividades bibliotecarias que perjudiquen al usuario y la biblioteca.

Todo lo anterior basado en una visión amplia y globalizada en beneficio de la biblioteca y usuario, una vez que se sabe que se pretende a futuro, se pueden detectar oportunidades e identificar proyectos o planes prioritarios. Para el alcance de los proyectos es necesario conocer a las personas más idóneas en gestionar y optimizar los diferentes recursos.

\section{Principios de la planeación}

La planeación se rige por una serie de principios cuya aplicación es indispensable para una mejor gestión de los planes o proyectos a implementar.

Factibilidad Los planes deben ser realizables, no tan ambiciosos, y que no sean imposibles de logar. Deben adaptarse a la realidad y los resultados a obtener.

Objetividad Basarse en datos reales, razonamientos precisos y exactos. Como estadísticas, estudios de mercado, estudios de factibilidad y datos numéricos. La cuantificación facilita la ejecución y la evaluación del progreso de los planes, es decir, el control.

Flexibilidad Al elaborar un plan siempre es conveniente establecer márgenes de holgura, que permitan afrontar situaciones imprevistas y que proporcionen nuevos cursos de acción, que se ajusten fácilmente a condiciones inesperadas.

Unidad Todos los planes específicos deben integrarse a un plan general, y 
dirigirse al logro de la filosofía organizacional, la visión, la misión y los objetivos de la organización, de tal manera que sean consistentes y armónicos en cuanto al equilibrio y la interrelación que deben existir entre todas las partes del plan y todas las áreas de la organización.

Del cambio de Cuando el plan enfrenta condiciones no previstas, ya sean oportunidades estrategias o amenazas, será necesario ajustarlo y crear estrategias alternas.

Fuente: Münch. L. (2008).p. 14-18

La planeación se da en todos los niveles directivos de la organización, la estratégica engloba a toda la organización es decir a la biblioteca en su conjunto, su característica es que realiza a largo plazo, va de 3 a 5 años en promedio, aunque puede ser más extenso, esto dependerá del tamaño de la organización. Esta fase pretende alcanzar la misión, objetivos organizacionales y planes o proyectos.

Dentro de la biblioteca el coordinador o jefe de biblioteca es el responsable de elaborar el plan estratégico, junto con los jefes de las distintas áreas que conforman la estructura organizacional del centro de información.

La planeación estratégica también conocida como administración estratégica o administración global, sin importar como la denominemos hay aspectos a considerar para que esta se lleve a cabo, tales como: diagnóstico, visión, misión, objetivos, estrategias, resultados y retroalimentación.

Los planes pueden fracasar por diversas razones:

- Que no se enfoquen al cumplimiento de la misión o a los objetivos generales.

- Que los objetivos no sean claros.

- Que el tiempo y los resultados planteados hayan sido mal estimados.

- No se controló, ni se dio seguimiento oportuno para identificar errores.

- Personal poco motivado para desempeñar bien los planes.

\section{Plan estratégico PE}

Actualmente dentro de las organizaciones, se trabaja en la realización de planes, ya sea estratégicos u operacionales, por plazo (corto o largo) y por 
frecuencia (únicos o permanentes). Los planes estratégicos son los que se aplican a toda la organización (en este caso la biblioteca), estos fijan las metas generales y tratan de posicionarla en su contexto; el cual abarca un plazo mayor y un panorama más amplio, esto va más allá de tres años (Robbins Stephen and Coulter, Mary 2005).

El responsable de biblioteca al elaborar el PE, es porque ya analizó la información y reflexionó el camino que debe emprender para lograr una biblioteca competitiva, innovadora, que busca mejorar cada día sus actividades, transformando los servicios para obtener la satisfacción plena de los usuarios. Lo anterior con el fin de consolidar el trabajo bibliotecario.

$\mathrm{El}$ punto central de un PE es diseñar los diferentes planes o proyectos que permitirán el logro de objetivos, misión y visión de la biblioteca, esto aunado a la visión, misión y objetivos generales de la institución a la que se esté subordinada.

Estos planes van de corto a largo plazo, lo que permite priorizar por tiempo, alcance o urgencia. Permiten un trabajo constante y direccionado, que se puede dar seguimiento e ir identificando desviaciones. Algo importante es que el personal de la biblioteca conozca los objetivos que persiguen y que sus actividades estén enfocadas a su realización de estos. Este instrumento de gestión debe estar por escrito y aprobado por las autoridades a las que se está subordinada y que tendrá la obligatoriedad de verse cumplido.

La estructura del plan de trabajo consiste en la creación de la filosofía institucional y un análisis del entorno externo e interno, cuyo propósito es conocer en qué situación se encuentra la biblioteca, para conocer las fortalezas y debilidades, siendo el último punto la definición de estrategias que dará pauta a la elaboración de planes y/o proyectos.

\section{¿Por qué hacer un plan estratégico?}

Es importante analizar y conocer el entorno donde se encuentra la biblioteca y sus pares. Con el avance de la misma disciplina, y el conocimiento de nuevas tecnologías, se tendrá del contexto de, quién soy, a dónde voy, cómo hacerlo, la probabilidad de lograr los objetivos y metas planteadas. 
$\mathrm{Si}$ se quiere elaborar un plan estratégico, se debe seguir la metodología del proceso administrativo, actualmente no existe un plan modelo, existen diversidad de planes, que van acordes a los requerimientos de las bibliotecas, a la experiencia de quien lo elabora y lo ambicioso que se pretende ser.

Se proponen en este trabajo algunos pasos indispensables para tener un plan estratégico, esperando sea guía para aquellos responsables de las bibliotecas que empiecen trabajar en la gestión bibliotecaria.

La biblioteca como cualquier organización, utiliza la administración para su buen funcionamiento, para esta actividad debe contar con la información por escrito o sistemas que ayuden a la recopilación, análisis y reporte de información.

La importancia de contar con un PE es:

- Planear en tiempo y forma lo que se va a realizar, para evitar planes improvisados o mal estimados.

- Definir objetivos, metas e indicadores para contrastar con los logros o avances.

- Priorizar planes para atender los urgentes e importantes.

- Cumplir con las necesidades y expectativas de los usuarios.

- Establecer mecanismos de control y de seguimiento.

- Optimizar recursos.

- Analizar situación real de la biblioteca, no dejando nada a la suposición.

Cuando las actividades o planes se realizan de manera espontánea o como van surgiendo se puede fracasar por diversas razones:

- Que no se enfoquen al cumplimiento de la misión o a los objetivos generales.

- Que los objetivos no sean claros o no se plantearon.

- Que el tiempo y resultados planteados hayan sido mal estimados o muy ambiciosos.

- No se controló, ni se dio seguimiento oportuno para identificar errores tanto al procedimiento como al personal. 
- Personal poco motivado para desempeñar bien los planes.

\section{Estructura del Plan estratégico}

Toda biblioteca debe tener un plan de trabajo por escrito para dar pauta al quehacer bibliotecario, donde se pueda visualizar los tiempos, los recursos y las personas que llevaran a cabo las acciones. Es importante tener un sistema de información donde se registren datos y avances para su análisis.

Se sugiere la siguiente estructura:

Tabla de contenido

Presentación o introducción

1. Filosofía institucional: misión, visión y valores

2. Objetivos generales

3. Diagnóstico FODA

4. Líneas estratégicas

5. Planes y/o proyectos

6. Control, Evaluación y Auditoría

7. Cronograma de trabajo

Anexos (tablas, cuadros)

A continuación, se describen de manera general los puntos antes mencionados

Presentación. Aquí se incluye una breve descripción sobre la biblioteca, logros y justificación sobre el trabajo a desarrollar y lo que persigue alcanzar.

Los puntos del uno al siete se abordarán en el apartado correspondiente.

Para elaborar el Plan es necesario hacerlo en tres fases (ver figura 1). El empleo del tiempo para cada fase dependerá del grado de análisis de la información, el plan estratégico deberá elaborarlo el responsable, coordinador o director de biblioteca lo antes posible a la toma del puesto. 


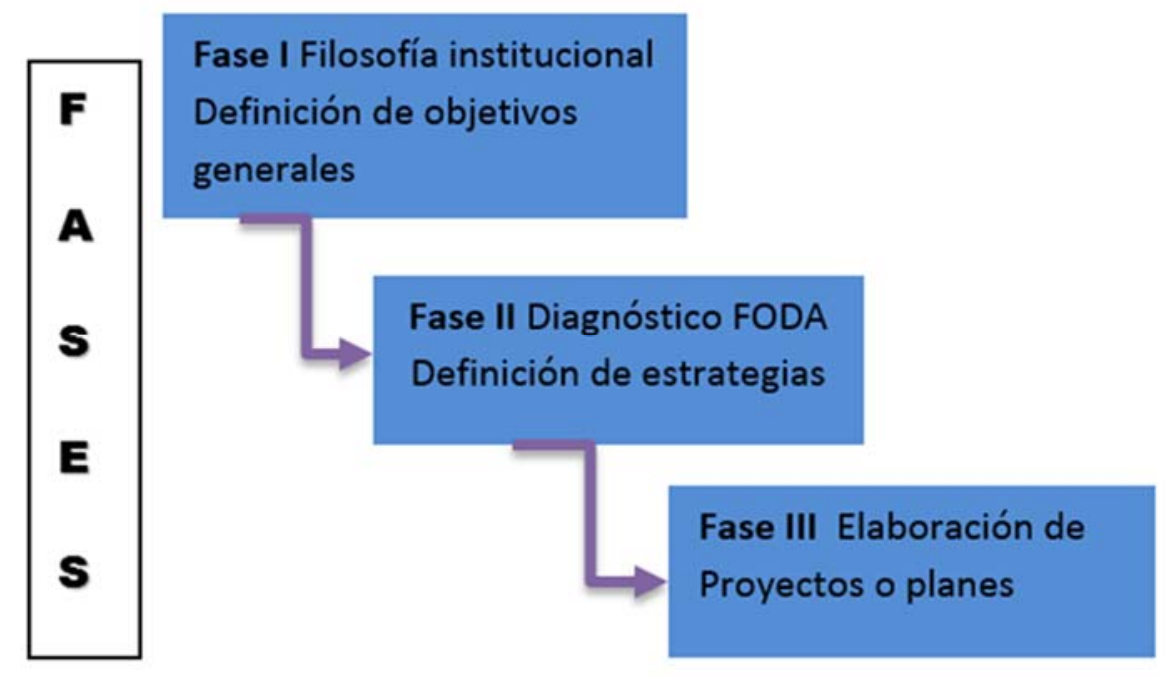

Figura 1. Fases del plan estratégico.

\subsection{Filosofía institucional}

La filosofía organizacional la define (Münch 2014) como “conjunto de postulados, valores, creencias y compromisos que rigen la vida de todos los integrantes de la organización". Para la generación de la filosofía, se requieren de tres elementos que son: la visión, la misión y los valores, algunas organizaciones incluyen el compromiso y el credo, cuando se cuenta con ella permite tener una identidad, de quiénes somos y que esperan de nosotros nuestros clientes/usuarios, esto incluye al mismo personal que labora y personas ajenas a la institución, pero que se relacionan con la biblioteca llamadas stakeholders, (proveedores, sindicato, bibliotecas, instituciones educativas, entre otros).

No se trata de tener una filosofía bien escrita, sino llevarla a cabo como tal, ya que será válida solo si se practica diariamente y se difundide a todo el personal de la organización.

Visión: Münch (Münch 2014a) describe "que es el estado deseado en el futuro. Provee dirección y forja el futuro de la organización estimulando acciones concretas en el presente". Es decir, la visión es una expresión verbal, de preferencia concisa, en ella se plasma lo que la biblioteca desea alcanzar en el futuro, debe ser ambiciosa pero alcanzable, algo que siempre marque la mejora continua, siendo inspiradora, donde el personal de la biblioteca se 
identifique, que pueda generar una cultura organizacional benéfica para la biblioteca. Como menciona Chiavenato (2010), la visión debe estar pensada en favorecer a todos los grupos de interés (stakeholders).

Misión: Es el motivo o la razón de ser por parte de una organización, una empresa o una institución, este se enfoca en el presente, es decir, es la actividad que justifica lo que la organización o el individuo está haciendo en un momento dado, responde a la pregunta ¿Cuál es el negocio de mi organización?, en el ambito bibliotecario, la pregunta sería ¿quiénes son mis usuarios y qué productos y servicios debo proporcionar?; la misión al redactarse debe considerar qué papel juega la biblioteca en el sector educativo y social, el valor que se desea lograr con la comunidad a la que se sirve y las actividades que se han de desempeñar para lograr lo anterior.

Valores: Los valores y creencias son abstractos, porque forman parte del actuar de las personas, dependerán qué tanto tengan arraigados estos. Cada empresa o lugar de trabajo forma su propia cultura organizacional, con base en lo que se pretende que actúe el personal.

Integridad y valores éticos: Son el resultado de las normas éticas y de conducta de la biblioteca. También forman parte de los proyectos que lleve a cabo el coordinador o director de la biblioteca para mitigar comportamientos impropios.

La importancia de que existan valores dentro de la biblioteca es con el fin de que todos los que trabajan en ella como los que asisten, los conozcan y se ejerzan como parte del actuar dentro del recinto. No se trata sólo de listar una serie de valores, sino que las personas se apropien y los apliquen de manera natural. La autoridad o nivel más alto de la biblioteca es quien los define y deben ser los primeros en ejercerlos ya que los valores ayudan a mejorar el clima laboral, a tener relaciones personales más cordiales, a un mejor trato de las autoridades con los subordinados y viceversa, así como con los usuarios. Los valores se relacionan con el tipo de organización, servicios y productos. Respecto a la biblioteca son esenciales los de servicio, innovación y calidad, pensando en los diferentes usuarios y que imagen queremos crear, como son la honestidad, comunicación y responsabilidad social, entre otros. Para definirlos, se debe hacer una lista de diferentes valores, analizarlos y 
seleccionar los más apropiados; priorizar por grado de importancia y por último definir cada uno de ellos, aunque sea de forma breve pero que aclare o se precise el actuar de cada uno de ellos, es importante mencionar que una vez definidos deben darse a conocer a todo el personal y a la comunidad.

\subsection{Objetivos generales}

Los objetivos generales que persigue la biblioteca deben estar planeados desde el punto de vista de logros, tiempo y modo, indican qué resultados se esperan a largo plazo. Estos dan la pauta a los diferentes planes y determinan las metas. Las diferentes áreas o departamentos que conforman la biblioteca deben seguir y apegarse a los objetivos generales, creando sus propios objetivos secundarios, siendo los tácticos o departamentales, que elaboran los responsables de cada área o sección.

\subsection{Análisis FODA (Fortalezas Oportunidades Debilidades y Amenazas)}

Para realizar el PE es imprescindible elaborar un análisis de la situación en la que se encuentra la biblioteca, por lo que es necesario, hacer un analisis FODA, para elaborar estrategias, formular objetivos y diseñar nuevos planes de trabajo, conocer la influencia y lo que sucede en cada entorno (ver figura 3), permitirá detectar los aspectos favorables y los desfavorables, lo que ayuda a planear, controlar y a tomar mejores decisiones.

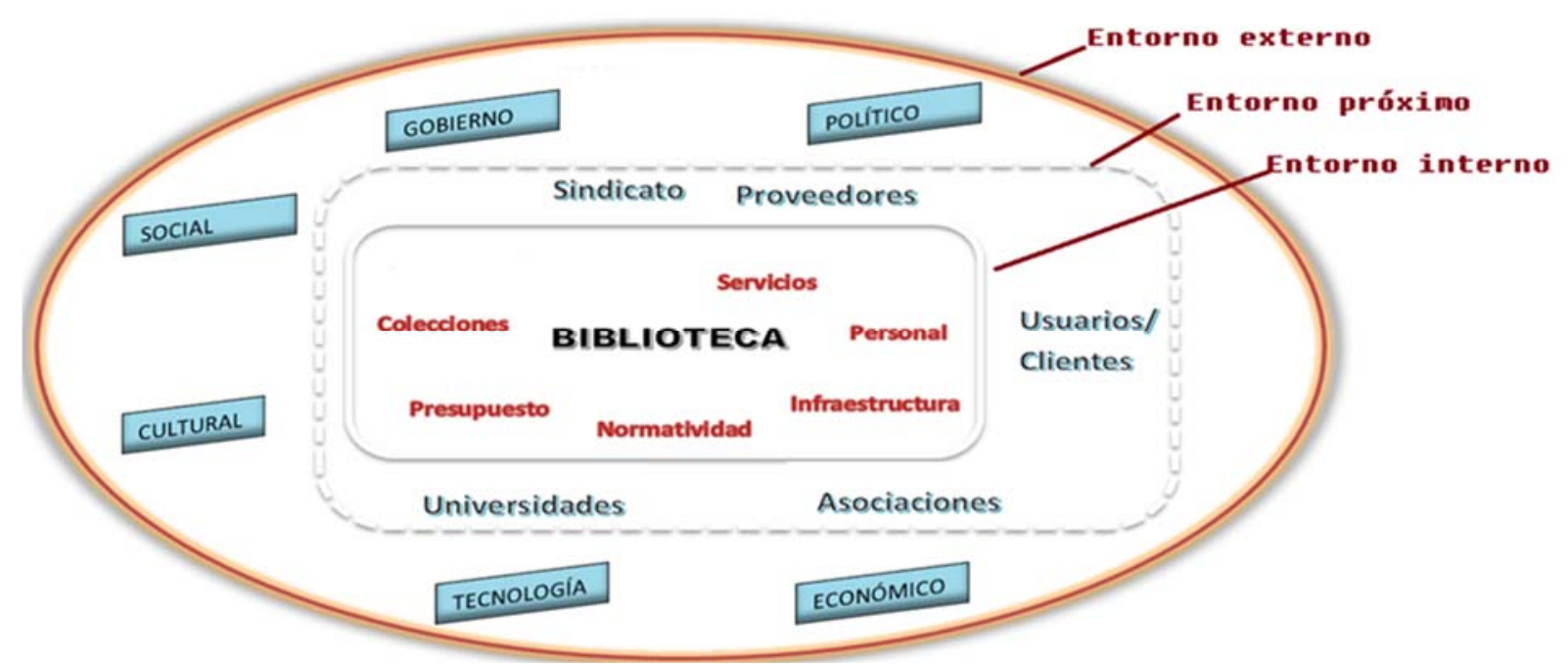

Figura 3 elaboración propia la biblioteca y su entorno 
Entorno interno. Habrá que evaluar los aspectos, que se traducen en los recursos de la biblioteca tanto tangibles como intangibles como: presupuesto, personal, conocimiento, innovación, infraestructura. Tendrá que verse internamente cómo se han hecho las cosas, si se cuenta con las habilidades, conocimiento y recursos necesarios.

Entorno externo. Es analizar o evaluar todos los aspectos que influyen en la biblioteca de manera positiva o negativa, ver tendencias, afectaciones, competencia, logros y avances. Estar al día en los avances tecnológicos, la economía, las nuevas políticas laborales, nuevos modelos educativos y todo aquello que influya a la toma de decisiones o desempeño de la biblioteca de manera directa o indirecta.

La organización debe tomar en cuenta las relaciones con las partes externas pero próximas, aquellas interesadas en la misma institución, hablando de bibliotecas serían: proveedores, sindicatos, universidades, asociaciones bibliotecarias, organizaciones culturales, bibliotecas afines y redes de bibliotecas. Ya que todos estos influyen en lo que hace la biblioteca y en cómo opera.

Realizar un análisis de los diferentes elementos requiere de estar informados, será más apegado a la realidad y no a supuestos. El valor de la información depende de dónde proviene, si es externa, que provenga de instituciones serias y reconocidas, la información interna, debe estar al día, sistematizada y a la mano.

Así mismo, los elementos a considerar dentro de personal: Políticas sobre el servicio, tareas y responsabilidades de cada puesto y área a la que se está suscrito, aptitudes y cualidades para el trabajo en equipo, competencias, comunicación, cultura y disponibilidad a nuevos cambios, contar con un programa de capacitación en relación a la cantidad de personal y a lo que se pretenda mejorar, los programas de higiene y seguridad (Olguin-Camacho y Hernández Galicia 2017) que aseguren la permanencia del personal, en condiciones favorables asegurando los recursos de la biblioteca, tanto humanos como materiales. 
Dentro de los aspectos tecnológicos: considerar las técnicas, procedimientos y métodos de trabajo utilizados. Estos pueden ser: equipo, productos, software, manuales. Sirven para incrementar la eficiencia, la racionalización y la especialización.

Los servicios son los productos que generamos y es importante analizar los atributos que estos tienen y cómo lo perciben o califican nuestros usuarios, saber si satisfacen necesidades o expectativas.

El presupuesto o recursos financieros son muy importantes ya que algunos proyectos dependen de ellos para realizarse y otros para mantenerse

La herramienta FODA ( $F$ fortalezas, O oportunidades, D debilidades y A amenazas) permite hacer el análisis de los entornos. Cada entorno tiene dos elementos:

\begin{tabular}{|lllll|}
\hline Entorno & Fortalezas & ¿En qué soy bueno? & Aprovecharlas y desarrollarlas. \\
& & ¿En qué me distingo? & Crear otras nuevas \\
\cline { 2 - 5 } & Amenazas & ¿Qué obstáculos hay? & Evitarlas o luchar contra ellas & \\
\hline Entorno & Oportunidades & ¿Qué puedo alcanzar? & Encontrarlas, aprovecharlas y \\
Externo & & Nuevas tendencias & explotarlas & \\
\cline { 2 - 5 } & Debilidades & Aquello que no hago & Evitarlas o disminuirlas \\
& & bien. ¿Qué debo evitar? & \\
\end{tabular}

Fuente: Miranda. Cómo elaborar un plan de empresa. $p 26$

Una vez que se ha realizado el análisis FODA, se detectan los puntos fuertes y débiles dentro y fuera de la biblioteca, que puede afectarla, se sabra entonces dónde se encuentra posicionada la biblioteca, esto dará pauta a elaborar las estrategias de aprovechamiento, de mejora, mantenimiento o de reforzamiento, que permitan avanzar en lo que esta debilitada, seguir haciendo bien lo que se hace, y/o replantearse si se cambian los planes o se ajustan.

\subsection{Líneas estratégicas}

Las estrategias son alternativas o cursos de acción, que muestran los medios y recursos que deben emplearse para lograr los objetivos (Münch 2014b). Son los diferentes caminos que puede haber para lograr un objetivo, se trata de visualizarlos para poder elegir el más idóneo. 
Al elaborarse las estrategias debe analizarse desde dos perspectivas, primero la externa, qué se tiene que hacer para seguir siendo competitivos, qué necesidades satisfacer de los diferentes usuarios, qué impacto se tiene en la sociedad, entre otros. Segundo, perspectiva interna, son todos los recursos con los que se cuenta, humanos, financieros y materiales.

$\mathrm{Al}$ diseñar las estrategias es importante considerar lo siguiente: analizar cada una de ellas en cuanto a su viabilidad, alcance y logro en tiempo, seleccionar cuidadosamente las mejores o las prioritarias. No olvidar que deben estar alineadas con la misión y objetivos. Cada una de ellas puede replantearse en nuevos proyectos o planes.

En algunos planes estratégicos se plantean enunciados breves y precisos, otros se colocan como las líneas estratégicas, para agrupar los planes y proyectos en la misma temática, existen diferentes tipos de estrategias a continuación se presentan algunas que pueden servir para su elaboración (Miranda 2004).

- Nivel de responsabilidad (segmentación)

- Relación con el entorno (crecimiento, estabilidad, defensiva)

- Relación con la competencia (líder)

A continuación, en qué consiste cada una de ellas:

Segmentación: Se refiere al nivel próximo de los usuarios, tiene que ver con la difusión de la biblioteca, fines o atributos de los productos o servicios, a quién se dirigen y cómo los van a percibir, conocer los factores que motivan a cada tipo de usuario en el uso de los recursos o servicios, es necesario conocer las necesidades satisfechas y los factores de insatisfacción.

Para conocer la opinión de los usuarios en cuanto a sus expectativas y satisfacción de necesidades, es necesario crear los canales de comunicación directos o no. El personal aquí juega un papel principal pues son quienes elaboran el servicio o producto.

Crecimiento: Es una estrategia con la que se trata de incrementar las actividades de la organización o acrecentando el número de servicios, tales como el 
crecimiento, en cuanto a ampliación de espacios físicos o fusión con otra biblioteca, por lo que la biblioteca debe aumentar sus ingresos, personal, alianzas y otras medidas cuantitativas.

Estabilidad: Se emplea para salvaguardar la posición actual, que prefiere mantener los números y resultados actuales, sin riesgos o en su caso, porque ya no se puede crecer más. Dentro de la biblioteca las decisiones de los responsables o autoridades tratan de asegurar que las actividades no sean alteradas por cambios a corto plazo.

Defensiva: Sirve para proteger todos los recursos de la organización con el objetivo de alcanzar alianzas para minimizar riesgos o reducir costos.

Lider. Se pretende ocupar una posición predominante frente a todos los demás sobre todo con bibliotecas pares y que así sea reconocida en el ámbito bibliotecario. Estableciendo un liderazgo a la comunidad que sirve, con calidad, innovación, difusión, recursos especializados o espacios modernos e incluyentes, el cual deberá marcar las pautas o tendencias a imitar por la competencia

\subsection{Planes y/o proyectos}

Hablar sobre los planes o proyectos que se pretenden realizar en un futuro, basado en la situación presente (diagnóstico), es parte de planeación estratégica, lo que permite visualizar lo posiblemente positivo y viable que asegure el bienestar de la biblioteca.

Un proyecto es un esfuerzo para organizar recursos humanos, materiales y financieros, logrando un alcance único en su campo de trabajo, con especificaciones definidas y con restricciones de costo y tiempo, de tal suerte que puede entregar o rendir cambios provechosos definidos por objetivos cuantitativos y cualitativos.

Los planes son los medios, a través de los cuales el administrador espera lograr los propósitos deseados. Es interesante cuestionarse algunas ideas para cuando se pretenden diseñar los nuevos planes, ya que estos se realizarán a futuro, esperando que las condiciones actuales no se alteren para el logro de 
estos. Un punto central es el usuario, si sus necesidades, comportamiento y expectativas serán las mismas o se habrán modificado, de esto dependerá la aceptación o satisfacción de los nuevos ofrecimientos bibliotecarios.

Cada uno de los planes que se elabore va ligado a cada una de las estrategias planteadas concretando así la planeación estratégica, un proyecto o plan se compone de: objetivos, acciones, plazos de inicio y fin, actividades generales o relevantes y asignación de los recursos que se necesitarán (Torres P. 215).

Debe considerarse la buena elección del personal que desempeñará los planes y coordinación de estos.

\subsection{Control, Evaluación y Auditoria}

La importancia de un sistema de control interno en la biblioteca es una garantía de lo descrito en el plan estratégico para asegurar lo planeado. La planeación y un sistema de control se complementan entre sí, porque mientras se determine una buena planeación y se incorporen controles efectivos dentro de la biblioteca, el resultado de ello será el aseguramiento de los bienes, logro de objetivos y seguir la misión de la biblioteca.

La evaluación es parte del proceso administrativo y es la última fase del ciclo, se evalúan los avances y resultados planteados en los diferentes planes o proyectos. Lo ideal es que se haya llevado a cabo de acuerdo con lo planeado, aunque no siempre resulta de esa manera, ya que en el transcurso pudieron haberse presentado algunas situaciones inesperadas que desvíen, detengan o modifiquen algún aspecto programado.

Chiavenato (2010) resume que "La planeación estratégica se diseña y ejecuta de forma que pueda ser evaluada, no sólo en términos de resultados finales, sino también en términos de comportamiento organizacional." Esto quiere decir que lo idóneo es ir controlando y evaluando las actividades de cada uno de los proyectos o planes establecidos, así será más fácil la detección de errores o desviaciones y no hacerlo cuando se han concluido los tiempos donde ya no pueda hacer nada. 
Cuando se planea se determinan y prevén objetivos, metas, estándares e indicadores. La función del control "consiste en asegurar que el desempeño real obtenga los resultados previamente deseados y planeados." (Chiavenato 2010a). Para ello, todos los involucrados deben conocer cuáles son los resultados esperados, motivando a que cada uno de lo mejor de sí para su cumplimiento, esto involucra: capacidades, habilidades, adaptación al cambio, motivación y estimación de los tiempos bien establecidos. Cuando se hacen las comparaciones de lo planeado o ejecutado con lo establecido, hay que determinar desde el principio los rangos de variación aceptables y los no aceptables, mismos que permiten que se acepten o no estas desviaciones.

Un objetivo del control no es controlar los comportamientos específicos, si no influir a través de su filosofía y valores en todo el personal de la biblioteca, para que así sus acciones y decisiones estén alineadas con las metas y objetivos de la biblioteca. "El objetivo principal del control consiste en indicar cuándo, cuánto, dónde y cómo se debe ejecutar la corrección necesaria y busca alcanzar dos fines:

- Corregir las fallas o errores existentes.

- Prevención de nuevas fallas o errores." (Chiavenato 2010b)

La biblioteca debería de llevar acabo auditorías dentro de los proyectos del plan estratégico, esto proporcionará información acerca de si el PE: se está realizando conforme a los criterios de la biblioteca, se está implementando y lo más importante si se está llevando a cabo de manera eficaz, eficiente y con economía.

Para esto es importante definir los criterios de la auditoría y el alcance de ésta, garantizando que la auditoría al PE se reporte al coordinador o director de la biblioteca. Una buena selección del personal interno o externo asegura la objetividad e imparcialidad en la auditoría.

\subsection{Cronograma de trabajo}

Una vez que se tienen los planes de trabajo bien definidos para un mejor control de los mismos respecto a los tiempos y su cumplimiento, se puede auxiliar de un cronograma, partiendo desde lo general hasta lo más específico. Los planes pueden tener su propio cronograma, por ejemplo, uno general que 
englobe todos los planes a llevarse a cabo en el periodo de gestión que se tenga, esto dependerá de la forma de trabajo de quien esté al frente de la biblioteca, es importante considerar dentro del cronograma algunos aspectos generales.

A continuación, se brinda un formato básico:

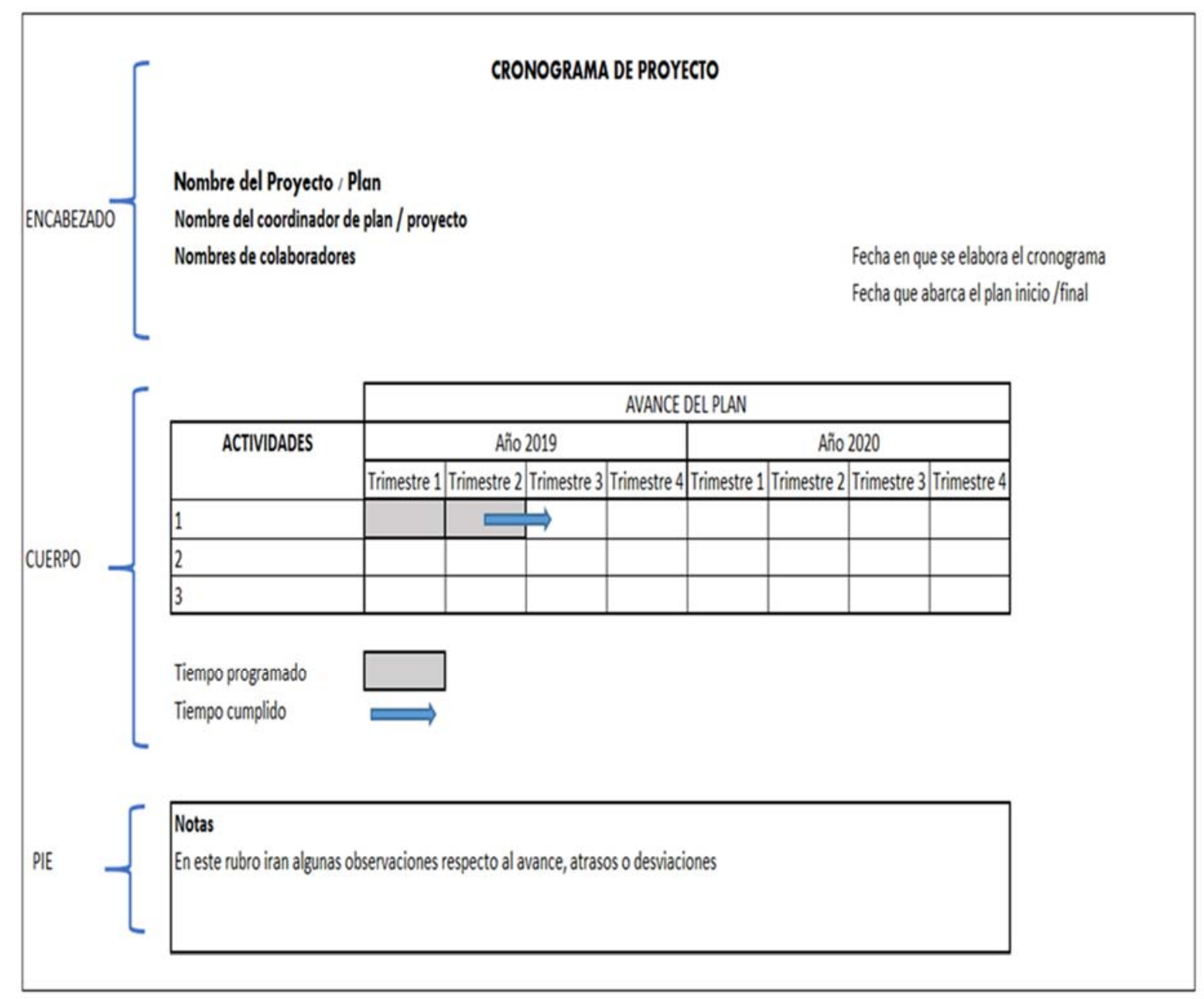

Anexos. Aquí se agrega todo aquello que puede hacer referencia al trabajo como tablas, cuadros o datos que complementan la información.

\section{Conclusiones}

Es necesario que toda biblioteca como organización se base en el proceso administrativo para elaborar un Plan Estratégico, este ayuda a que los directores, coordinadores o jefes de la bibliotecas y/o centros de información 
de una organización, plasmen la dirección a seguir de la institución o empresa, y cuando este plan es transmitido de manera eficiente hacia toda la organización, ésta generará correlaciones en todo el personal que labora en la biblioteca para la obtención de sus objetivos y cumplir con la misión que es la esencia de dicha organización.

Asimismo, el plan ayuda a que cada trabajador sepa hacia a donde se quiere ir y se comprometa con ese destino. Como primera fase es esencial que la administración, gestión, planeación deben llevarse a cabo de manera incipiente, delimitando así el rumbo a seguir en los mejores tiempos y optimización de los recursos humanos, materiales y financieros con que cuente la biblioteca.

Aunado a lo anterior, se plantean estrategias de acuerdo con la situación en la que se encuentre nuestra biblioteca, definiendo el rumbo a seguir y consecución de los objetivos, sin dejar atrás la fase de control, teniendo en cuenta la importancia de verificar lo establecido en los tiempos acordados.

Lo que se espera con dicho trabajo es ayudar a mejorar la labor bibliotecaria evitando planes o trabajos improvisados, planteando acciones a emprender, basándose en la investigación, análisis y estudio de los mismos. Para ello es necesario que se conozcan los aspectos básicos del proceso administrativo y de las herramientas que enriquecen cada una de las fases.

En la medida que se pongan en marcha fases del proceso administrativo, el director o jefe de biblioteca podrá ser competente para tener directrices claras, para sus subordinados y colaboradores con lo que se busca el compromiso de cada integrante de la organización.

El contar con un proceso de Planeamiento Estratégico ayuda a la formulación de objetivos y estrategias alineados hacia una gran meta en común. Más allá de establecer Definiciones Institucionales como la Misión, Visión y Valores.

Un Plan Estratégico debe indicar las pautas a seguir de la biblioteca para la gestión de un período determinado. No bastará con establecer objetivos claros y participativos, sino saber cómo se llevarán a cabo en las estrategias para alcanzarlos. 


\section{Bibliografía}

AGUILAR CRUZ, Conrado, 2000. La necesidad de la planeación estratégica en las organizaciones industriales modernas. Temas de ciencia y tecnología, 4(11), 17-28 [ Consultado 12 febrero 2019] Disponible en: http://www.utm.mx/temas/temasdocs/e1117.pdf

CASAGRANDE, Renato, 2002. Valores organizacionales: un análisis en el contexto educativo. Buenos Aires: UNESCO. [consultado 05 enero 2019]. Disponible en: http://unesdoc.unesco.org/images/0013/001321/132158s.pdf

CASTILLO, Diana Araceli, 2012. El pensamiento estratégico como herramienta para la elaboración de planes de trabajo. Tesis UNAM. [ Consultado 18 diciembre de 2018] https://es.scribd.com/document/349146589/EL-PENSAMIENTOESTRATEGICO-COMO-HERRAMIENTA-PARA-LA-ELABORACION-DEPLANES-DE-TRABAJO-pdf

CHIAVENATO, Idalberto, 2010. Planeación estratégica. 2ª ed. México: McGraw Hill. p. 318. ISBN 9789701068779.

Directrices IFLA / UNESCO para el desarrollo del servicio de bibliotecas públicas, 2001. México [ Consultado 25 enero 2019] https://www.ifla.org/files/assets/hq/publications/archive/the-public-libraryservice/pg01-s.pdf

FUSHIMI, Marcela, 2003. Autoevaluación de bibliotecas universitarias: Modelo de medición y diagnóstico a partir de datos estadísticos. Tesis http://www.memoria.fahce.unlp.edu.ar/tesis/te.294/te.294.pdf

ISLAS, Octavio, 2011. Importancia de la filosofía institucional [Consultado 27 mayo 2019] https://octavioislas.files.wordpress.com/2011/05/filosofc3ada-institucional.pdf

MIRANDA OLIVÁN, Antonio, 2004. Cómo elaborar un plan de empresa. Madrid: Thomson, p.174, ISBN 9788497323253

MÜNCH, Lourdes, 2008. Planeación estratégica: el rumbo hacia el éxito. México: Trillas. 126 p.

MÜNCH, Lourdes, 2014. Administración: gestión organizacional, enfoques y proceso administrativo. $2^{\mathrm{a}}$ ed. México: Pearson, 336 p.

MURILLO, Javier, 2012. ¿Cómo hacer un plan de trabajo? La Metodología Paso a Paso. [Consultado noviembre del 2018] http://axeleratum.com/2012/icomo-hacer-unplan-de-trabajo-paso-a-paso-de-la-metodologia

OLGUIN-CAMACHO, Cesar S. y HERNADEZ GALICIA, Elsa, 2017. Normas básicas de seguridad y salud para edificios de biblioteca según las Normas Mexicanas 
(NOM). Bibliotecas, 35(1), 1-19. EISSN: 1659-3286 [Consultado septiembre del 2019]. https://doi.org/10.15359/rb.35-1.2

ROBBINS,Stephen and COULTER, Mary, 2005. Administración. $8^{\mathrm{a}}$ ed. México: Pearson. Electronic books, p. 642, ISBN 9702614619.

RODRÍGUEZ VALENCIA, Joaquín, 2002. Como aplicar la planeación estratégica a la pequeña y mediana empresas. 5 a ed. México: Thompson, p.339.

SAINZ DE VICUÑA, Ancín, José María, 2012. El plan estratégico en la práctica. $2^{\mathrm{a}}$ ed. México: Omega, p.391, ISBN 9786077074120.

TERRY, George R., 1971. Principios de administración. México: CECSA, p. 879, ISBN 970554539.

TORRES HERNÁNDEZ, Zacarías, 2014. Planeación y Control: una visión integral de la administración. México: Patria. 400 p.

UNIVERSIDAD COMPÚTENSE DE MADRID, 2015. Plan Estratégico de la Biblioteca

Complutense 2014-2016. [Consultado 28 febrero 2019]

https://biblioteca.ucm.es/data/cont/docs/60-2015-01-26-

PLAN\%20ESTRATEGICO \%20enero\%202015.pdf 